\title{
Reduced-Dispersion Duplex DQPSK Radio-Over-Fiber Communications Using Single-Laser-Based Multiple Side-Bands
}

\author{
Salman Ghafoor and Lajos Hanzo \\ School of ECS \\ University of Southampton, SO17 1BJ, UK. \\ Email: saglz07@ecs.soton.ac.uk, lh@ecs.soton.ac.uk
}

\begin{abstract}
Multiple side-bands are generated from a single laser diode with the aid of optical carrier suppression, which facilitates the transmission of reduced-rate parallel streams mapped to the side-bands. As a benefit, all the optical signal processing tasks, including demultiplexing of a high-rate signal into multiple low-rate signals can be performed at the central unit. The technique can also be useful in reducing the effects of dispersion in case of high-frequency Radio Over Fiber (ROF)communications by transmitting a single-data rate signal as multiple low-rate signals using the side-bands generated. We demonstrate the duplex transmission of a bit-rate of $\mathbf{7 6 8}$ Mbit/s over a $50 \mathrm{~km}$ fiber section using three side-bands in each direction. The multiple side-band aided system has a BER performance, which is close to that of the idealized benchmarker operating in a back-to-back mode.
\end{abstract}

\section{INTRODUCTION}

Radio over fibre systems constitute a cost effective solution for the interconnection of Base Stations (BS) or for feeding the so-called distributed antennas [1]. The BS typically employs a Photo-Detector (PD) for detecting the signals of other BSs received through the Mobile Switching Center (MSC). The detected signal is then remodulated, amplified by a Radio Frequency (RF) amplifier and then transmitted to the mobiles. A range of different methods designed for increasing the effective transmission distance of ROF links have been studied for example in [2] and [3]. In [2], it has been shown that the effective optical transmission distance can be beneficially increased by overcoming the effects of dispersion, for example by using Single Side-Band (SSB) modulation with the aid of a dual-drive external modulator. Another technique of mitigating the fiber-induced dispersion invokes predistortion at the transmitter using passive filters along with a suitable modulation scheme [3]. The typical MSC-BS links are duplex and the two directions may use a different frequency band. However, the employment of a single light source in the ROF system for both directions constitutes an attractive design option, since it eliminates the need for two separate light sources at the MSC linking, for example $\mathrm{BS} 1$ and BS2. In order to achieve this desirable objective, numerous schemes have been proposed [4], [5], [6] and [7]. In [4], [5] and [6], the light-wave carrier used in the MSC-toBS1 link of Fig 1 for example may also be used in the BS1to-MSC transmission by modulating the same carrier using different modulating formats in the two directions. In [7], the light-wave signal generated at the MSC is passed through a Mach-Zehnder modulator (MZM) and modulated by a RF signal to generate two optical side-bands centered around the optical carrier frequency. The optical carrier is suppressed at the output of the MZM and each of the side-bands is used for transmission to different BSs under the control of a specific MSC. This technique of generating multiple sidebands, while suppressing the carrier is referred to as Optical Carrier Suppression (OCS) [8], [9] and [10].

Again the ROF system's architecture considered is shown in Fig 1, where a Public Switched Telephone Network (PSTN) delievers the tele-traffic to the MSC using an optical fiber. The MSC then routes the tele-traffic from the PSTN to the appropriate BSs in its vicinity. Finally, the BSs forward the corresponding RF signal to the Mobile Stations (MS). Our goal is to design a single-diode aided OCS transmitter-receiver pair (transceiver) for employment either at the MSCs or BSs.

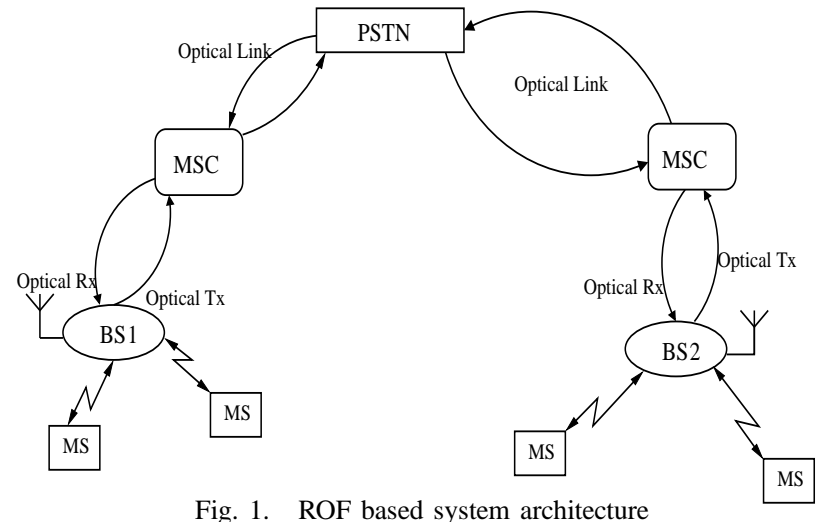

Against this background the novel contribution of the paper is:

1- Generation of multiple optical side-bands using a single laser source for parallel transmission of reducedrate RF signals. The multiple RF signals can be processed individually at the MSC and transmitted to the same or different BSs enabling the ROF system to perform all the complex signal processing tasks at the MSC. The paper also suggests a duplex architecture that does not need separate optical sources at the BSs for the uplink transmission of multiple RF signals.

2- The technique can also be helpful in reducing the dispersion-induced distortions of high-rate signals transmitted at high electrical carrier frequencies, such as for example at $60 \mathrm{GHz}$. These high-rate signals 
when transmitted as multiple low-rate signals will undergo reduced dispersion-induced distortions.

The outline of the paper is as follows. In Section 2, we discuss the OCS technique, which generates parallel higher order side-bands from a single laser source. Section 3 details the proposed bi-directional link, bearing in mind the ROF architecture of Fig 1. In Section 4 we provide our numerical results for the duplex MSC-BS transmission of ROF signals. The simulation tool used for our numerical study is the commercial software VPITransmissionMaker 8.5 (www.vpiphotonics.com). Finally, Section 5 offers our conclusions.

\section{Generating Multiple Side-BAnds using OCS}

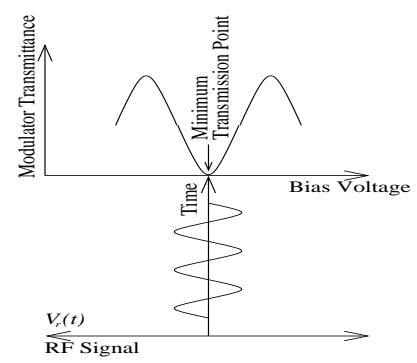

(a)

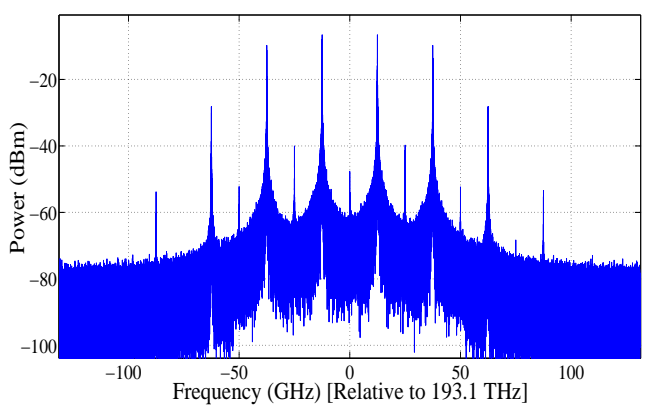

(b)

Fig. 2. (a). Time-domain operation of the OCS. (b). Optical spectrum at the output of MZM

To elaborate further in the context of Fig 2, we use the OCS technique to generate six optical side-bands from a single laser source using a Dual-Drive MZM (DD-MZM). We observe from Fig 2.a that the MZM is biased to a non-zero DC value. At this point a sinusoidal RF signal is superimposed on the DC bias in order to generate an optical signal, whose carrier is suppressed. The optical carrier of $E_{c}(t)=A_{c} \exp \left(j w_{c} t\right)$ generated by the Laser Diode (LD) having an amplitude of $A_{c}$ and a frequency of $w_{c}$ is applied to the input of the DD-MZM. The two arms of the MZM are driven by sinusoidal RF signals having equal amplitudes and a $180^{\circ}$ phase shift. The two drive voltages applied to the MZM's electrodes can be written as $V_{r 1}(t)=V_{d c 1}+V_{r} \cos \left(w_{r} t+\phi_{1}\right)$ and $V_{r 2}(t)=V_{d c 2}+$ $V_{r} \cos \left(w_{r} t+\phi_{2}\right)$ where $V_{d c}, V_{r}, w_{r}$ and $\phi$ are the DC bias voltages, RF signal amplitude, RF signal frequency and phase, respectively. As seen in Fig 2.a, the MZM's transmittance versus bias characteristic is a nonlinear function, therefore it will generate higher order optical side-bands, when a RF signal is applied to its terminals. The number of higher order side-bands generated depends on the RF signal's amplitude $V_{r}$. As seen in Fig 2.a, a high value of $V_{r}$ increases the nonlinearity of the MZM's transfer characteristic, which results in an increased number of higher-order side-bands generated at the output. The optical signal at the output of the DD-MZM may be written as the sum of the optical signal in each arm of the MZM as below:

$$
E_{M}(t)=\frac{A_{c}}{2}\left(e^{j \frac{\pi V_{r 1}(t)}{V_{\pi}}}+\gamma e^{j \frac{\pi V_{r 2}(t)}{V_{\pi}}}\right) e^{j w_{c} t},
$$

where $V_{\pi}$ is the voltage required to induce a phase-shift of $\pi$ for each of the MZM arms and $\gamma$ accounts for the non-ideal extinction ratio $\varepsilon$ of the MZM. The value of $\gamma$ lies between 0 and 1 , which is related to $\varepsilon$ as $\gamma=(\sqrt{\varepsilon}-1) /(\sqrt{\varepsilon}+1)$. The output of the DD-MZM may be further expressed in terms of Bessel functions as [11]:

$$
\begin{aligned}
E_{M}(t) & =\frac{A_{c}}{2}\left[\sum _ { n = - \infty } ^ { \infty } J _ { n } ( m _ { r } ) \left(e^{j\left(\phi_{v}+n \phi_{d}\right)}\right.\right. \\
& \left.\left.+\gamma e^{-j\left(\phi_{v}+n \phi_{d}\right)}\right) e^{j n\left(w_{r} t+\phi_{m}\right)}\right] \cdot e^{j w_{c} t},
\end{aligned}
$$

where $J_{n}$ is the Bessel function of first kind and order $\mathrm{n}$, and

$$
\begin{aligned}
m_{r} & =\frac{\pi V_{r}}{V_{\pi}} \\
\phi_{v} & =\frac{\pi\left(V_{d c 1}-V_{d c 2}\right)}{2 V_{\pi}} \\
\phi_{d} & =\frac{\left(\phi_{1}-\phi_{2}\right)}{2} \\
\phi_{m} & =\frac{\left(\phi_{1}+\phi_{2}\right)}{2} .
\end{aligned}
$$

The value of $V_{r}$ chosen in our simulations is $0.9 \cdot V_{\pi}$, therefore the argument of the Bessel function $m_{r}$ becomes 2.82. Furthermore, in order to operate the DD-MZM in a double side-band suppressed carrier (DSB-SC) configuration, we choose $\phi_{1}-\phi_{2}=\pi$ and $V_{d c 1}-V_{d c 2}=V_{\pi}$. Substituting values in Equation 2 and simplifying it gives the following relation for the optical signal at the output of the DD-MZM:

$$
\begin{aligned}
E_{M}(t) & =\frac{A_{c}}{2}\left[\sum _ { n = - \infty } ^ { \infty } J _ { n } ( 2 . 8 2 ) \left(j\left(e^{j n \pi}-\gamma\right)\right.\right. \\
& \left.\left.\times e^{j n\left(w_{r} t+\phi_{1}\right)}\right)\right] \cdot e^{j w_{c} t}
\end{aligned}
$$

It can be observed from Bessel function expansion of Equation 4 that for an argument of 2.82 , we should obtain nearly six side-bands on each side of the input optical carrier. The power of each side-band depends upon the value of the extinction ratio $\varepsilon$ of the MZM and can be calculated using Equation 4. Equation 4 suggests that a high value of $\varepsilon$ will result in a higher suppression of both the carrier and of the even-indexed side-bands.

The spectrum of the OCS signal generated in our simulations is shown in Fig 2.b. This spectrum was obtained by 


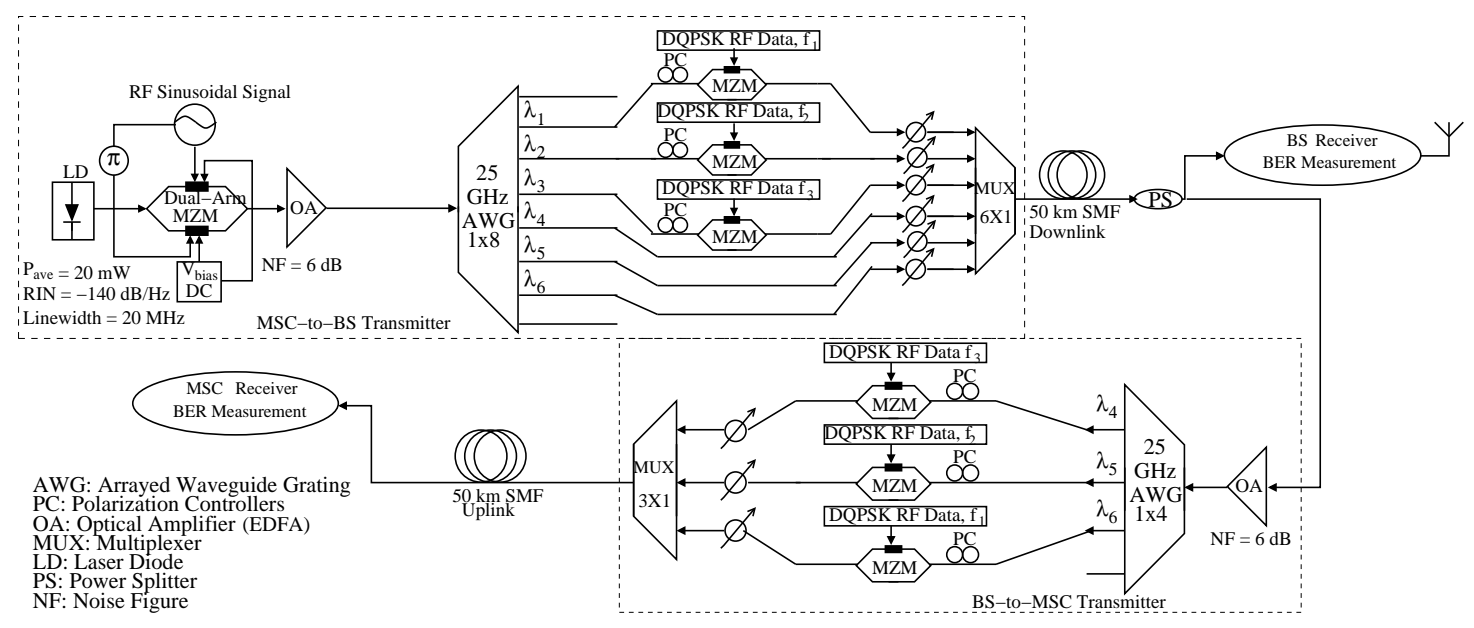

Fig. 3. Schematic of the proposed bi-directional optical link

applying a RF signal having a frequency of $12.5 \mathrm{GHz}$. It can be observed from Fig 2.b that the output spectrum of the MZM contains a carrier attenuated by $40 \mathrm{~dB}$, while the even-indexed side-bands are attenuated by $32 \mathrm{~dB}$ compared to the first-order odd-indexed side-bands. The frequency spacing between the supressed carrier and the first side-band on both sides of the carrier as well as that between the consecutive odd-indexed side-bands is twice that of the RF frequency applied. The OCS technique allows us to obtain six different-wavelength optical carriers from a single laser source. Therefore this technique is useful in terms of reducing both the cost and complexity of a MSC or BS, which would require six differentwavelength laser sources in the absence of the proposed OCS technique. Furthermore, the OCS technique allows us to accurately control the spacing between the side-bands by simply varying the frequency of the RF signal applied.

\section{THE PROPOSED BI-DIRECTIONAL LINK}

The schematic of our system is shown in Fig 3. The signal generated by the laser diode (LD) having the parameters shown in the figure is passed through a DD-MZM biased using a DC source. A RF sinusoidal signal having a frequency of $12.5 \mathrm{GHz}$ is applied to the upper and lower branches of the MZM with the aim of generating multiple side-bands at the desired frequencies, as seen in Fig 2.b. The signal at the output of the MZM consists of higher-order side-bands, again as shown in Fig 2.b. This signal is then amplified using an Erbium Doped Fiber Amplifier (EDFA) having a noise figure of $6 \mathrm{~dB}$. The output of the EDFA is fed into a 1x8 Arrayed Waveguide Grating (AWG) which has a channel spacing of 25 GHz. The AWG has parameters similar to the $25 \mathrm{GHz}$ AWG manufactured by ANDevices. The transfer function of AWG's filtering exhibits a Gaussian shape having a $3 \mathrm{~dB}$ bandwidth of $25 \mathrm{GHz}$. At the output of the AWG, the six desired oddindexed side-bands spaced $25 \mathrm{GHz}$ apart are retrieved. The optical signals having different central frequencies are labelled by $\lambda_{1}, \lambda_{2}, \lambda_{3}, \lambda_{4}, \lambda_{5}$ and $\lambda_{6}$, as shown in Fig 3 . The signals $\lambda_{1}, \lambda_{2}$ and $\lambda_{3}$ used for example for the MSC-BS downstream transmission, are intensity modulated at the MSC using a
MZM by the RF DQPSK signals having central frequencies of $f_{1}, f_{2}$ and $f_{3}$, respectively. After intensity modulation, the resultant ROF signals having optical carriers of $\lambda_{1}, \lambda_{2}$ and $\lambda_{3}$ are attenuated and multiplexed with the remaining three signals, namely with $\lambda_{4}, \lambda_{5}$ and $\lambda_{6}$, which are then transmitted without imposing any modulation, using the MUX block of Fig 3. The signals $\lambda_{4}, \lambda_{5}$ and $\lambda_{6}$, which will be used later for upstream transmission at the BSs, are also attenuated to suitable levels before being multiplexed at the MSC. Since the EDFAs amplify all the six optical side-bands together, it has no individual control over the power of each side-band. The attenuators are used for adjusting the required input power of each optical side-band. The symbol rate of the DQPSK RF signal is $128 \mathrm{Msymbols} / \mathrm{s}$, hence a data rate of $256 \mathrm{MBit} / \mathrm{s}$ is supported. The central frequencies $f_{1}, f_{2}$ and $f_{3}$ of the RF signals are $1.792 \mathrm{GHz}, 2.048 \mathrm{GHz}$ and 2.304 $\mathrm{GHz}$ respectively. As mentioned in [2] and [12], a dispersive fibre exhibits a frequency dependent transfer function. The relationship between the received power $P_{e l}$ of the electrical signal for a fibre having a length of $L$ and a dispersion parameter $D$ is given by [12],

$$
P_{e l} \propto \cos \left[\pi L c D\left(\frac{f_{e l}}{f_{o}}\right)^{2}\right],
$$

where $c$ is the speed of light, $f_{e l}$ is the frequency of the electronic signal and $f_{o}$ is the central frequency of the optical carrier. It can be deduced from Equation 2 that the quality of the received RF signals at the receiver will be affected depending upon their central frequencies. Furthermore, since we have three unmodulated continous wave signals along with the modulated signals travelling in the downstream direction, Cross Phase Modulation (XPM) will be encountered between the signals. The XPM imposes phase noise which is converted to amplitude noise by the dispersive fiber [13]. The phase noise increases upon increasing the average power of the signal, therefore the average power per optical sideband should be kept low. In order to minimize the XPM as well as the distortions produced by Self Phase Modulation (SPM), we have used the optical attenuators shown in Fig 
3 to reduce the optical power per channel. The power of each optical channel in our simulation is reduced to $-1 \mathrm{dBm}$ before transmission. Following optical multiplexing in Fig 3, the signals are transmitted over a $50 \mathrm{~km}$ Standard Single Mode Fiber (SSMF) section. It is worth mentioning here that we can extract any of the signals $\lambda_{1}-\lambda_{3}$ at a shorter length of fiber to deliver RF signals to BSs at different distances. However, in our investigations we have considered the worst-case scenario, where all the signals propagate along the entire $50 \mathrm{~km}$ section of SSMF. After travelling through the SSMF, the signal is split into two paths, one of which goes to the BS receiver, while the other to the BS transmitter. At the BS receiver, a 1x4 AWG having the same channel-spacing and filtering parameters as the one used at the transmitter, separates the three modulated side-bands. At the BS transmitter, again a 1x4 AWG separates the unmodulated optical side-bands $\lambda_{4}$ $\lambda_{6}$. In the absence of these unmodulated signals $\lambda_{4}-\lambda_{6}$, the BSs would require separate LDs to transmitt the upstream signal back to the MSC's receiver. The filtered signals $\lambda_{4}$ $\lambda_{6}$ generated at the output of the $1 \times 4$ AWG seen in Fig 3 are then intensity modulated by the RF DQPSK signals to be transmitted from the BS to the MSC, which have central frequencies of $f_{3}, f_{2}$ and $f_{1}$, respectively. We have considered the same data rate and modulation format for the upstream BS-MSC RF signals as the downstream MSC-BS RF signals. The ROF signals are then optically multiplexed in Fig 3 and transmitted over another $50 \mathrm{~km}$ fiber section to the MSC's receiver. After propagating through the fiber, the signals are received at the MSC and detected as seen in Fig 3. In order to quantify the integrity of the received signals, we have used a RF DQPSK receiver at the MSC. The architecture of the MSC receiver has elements reminiscent of the BS's receiver and is shown in Fig 4. More explicitly, the MSC receiver's input has a $1 \mathrm{x} 4 \mathrm{AWG}$ to extract the required optical signals, say $\lambda_{x}$. The optical signal is then detected by a PD, whose parameters are also shown in Fig 4.

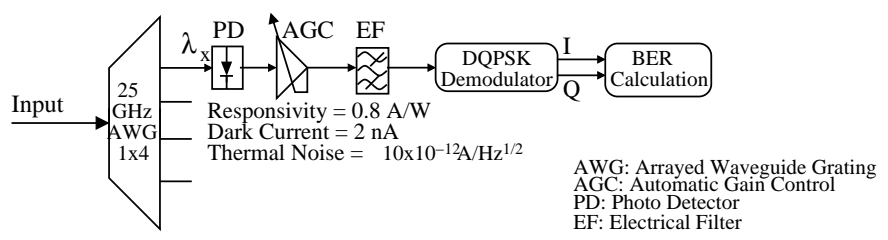

Fig. 4. DQPSK receiver setup

The resultant electronic RF signal at the output of the PD of Fig 4 also contains the upper harmonics of the desired signal, which are generated by the non-linear process of photodetection. As seen in Fig 4, this electronic signal is then amplified using an electronic Automatic Gain Control (AGC) amplifier. The parameters of the AGC were configured for maintaining a constant output power for variable input RF powers. After AGC-aided amplification, the signal is passed through a first order Gaussian Electronic Filter (EF), which is a band-pass filter having an identical center frequency to that of the RF signal and a $3 \mathrm{~dB}$ bandwidth of five times the symbol rate. The signal at the output of EF is then demodulated by the DQPSK demodulator of Fig 4, as detailed in [14]. The lowpass filters of the demodulator seen in fig 4 exhibit a Gaussian shape having a $3 \mathrm{~dB}$ bandwidth of 0.85 times the symbol rate. The ON-OFF keyed I and Q electrical bit sequences at the output of the DQPSK demodulator are then subjected to BER calculation in Fig 4 for the I and Q signals separately. The BER of the DQPSK signal is obtained by averaging the BERs of the I and Q signals [15].

\section{Performance Results}

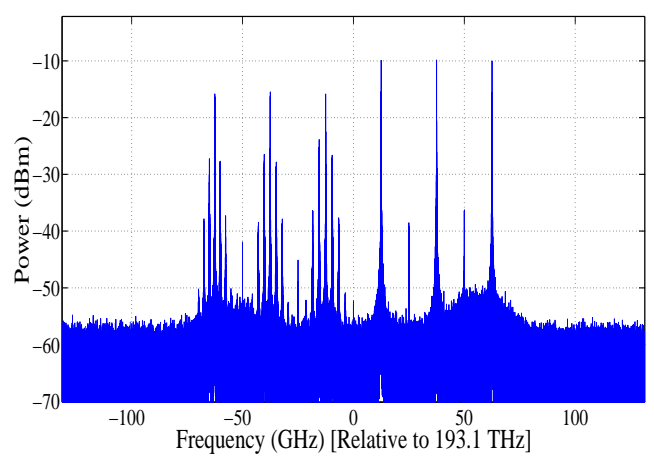

(a)

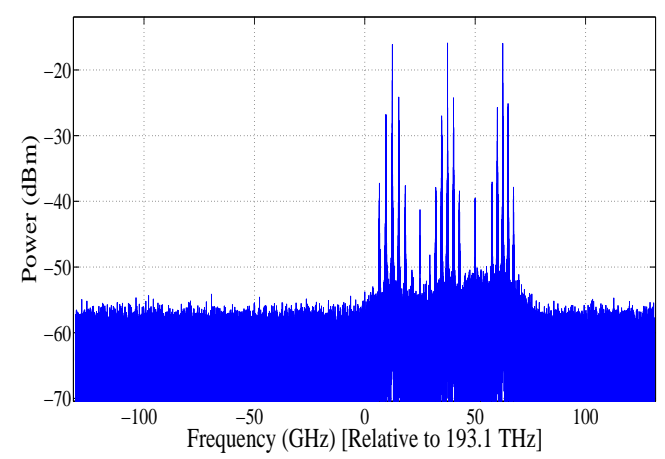

(b)

Fig. 5. (a). Spectrum of the Downstream Input Signal of the Fiber. (b). Spectrum of the Upstream Input Signal of the Fiber.

Let us now characterize the proposed architecture's performance. Fig 5.a and Fig 5.b show the optical spectrum of the ROF signal at the input of the fiber in the MSC-BS downstream and in the BS-MSC upstream directions, respectively. As seen in Fig 5.a, the MSC-BS downstream signal spectrum consists of six optical side-bands. Three of these carriers are intensity-modulated to generate the ODSB signals, while the signals $\lambda_{4}-\lambda_{6}$ are transmitted without any modulation. Fig 5.b shows the BS-MSC upstream signal spectrum at the input of the fiber. It can be observed from Fig 5.a and 5.b that there is a slightly higher degree of spectral noise at the base of the higher-order side-bands compared to the first-order side-band. The reason for this is the lower powers of the higher-order side-bands at the output of the DD-MZM, which generates a higher ASE noise at these wavelengths in the EDFA. The BER performance of the downstream and upstream is characterized in Fig 6.a and 6.b, respectively. In Fig 6, we 
have also presented the BER measurements for the back-toback benchmarker by removing the SSMF from the setup. Observe in Fig 6 that despite the $50 \mathrm{~km}$ length of the SSMF, the BERs of both the upstream and downstream signals are quite low even at low received optical powers. A comparison between the BERs of the downstream and upstream signals with their respective back-to-back measurements reveals that for some channels, the BERs recorded for the SSMF are lower than the back-to-back BERs. This behaviour may be attributed to the more substantial power attenuation of the IMD components compared to the attenuation of the RF signal's power while travelling in the fiber [16]. It has been shown in [16] that for an optimum input power of the RF signal, the IM3 distortion imposed by the MZM decreases in power faster than the signal power, hence resulting in an increased Signal to Noise and Distortion Ratio (SNDR) for the system. However, further investigations are required to characterize this effect in more depth for the proposed system. It is worth mentioning here that the different components used in our system have parameter values that are similar to the characteristics of practical components used in RF and optical communication systems.

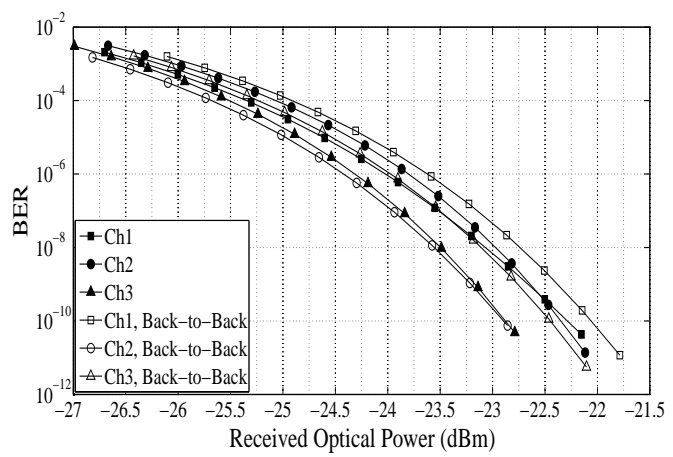

(a)

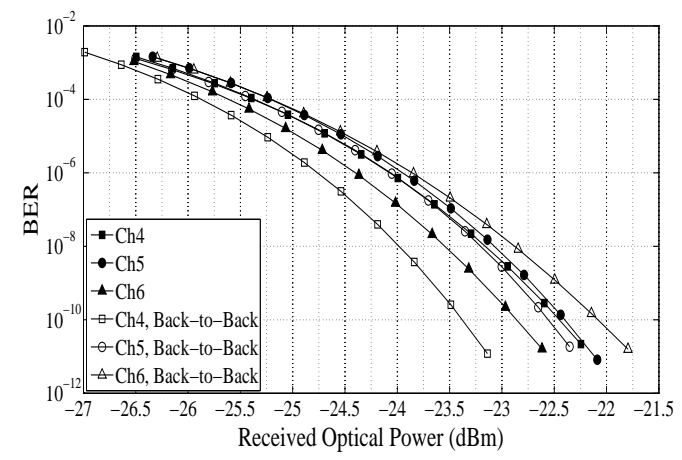

(b)

Fig. 6. BER versus received power performance in the (a) MSC-BS downstream and in the (b) BS-MSC upstream directions

\section{Conclusions}

A flexible bi-directional/duplex optical transceiver was designed using a single LD, which is capable of splitting a high-rate stream into reduced-rate parallel streams, hence rendering the data less susceptible to fiber-induced dispersion.
The parallel streams are mapped to a number of optical sidebands. Despite using a $50 \mathrm{~km}$ SSMF section, the system performs close to its idealized back-to-back benchmarker, which encounters no fibre induced-dispersion. Our future research will consider the performance of higher-throughput modulation schemes and optical OFDM in comparison to the fiber's information theoretic capacity.

\section{REFERENCES}

[1] A. Hekkala, M. Lasanen, I. Harjula, L. C. Vieira, N. J. Gomes, A. Nkansah, S. Bittner, F. Diehm, and V. Kotzsch, "Analysis Of And Compensation For Non-Ideal ROF Links In DAS," IEEE Wireless Communications, vol. 17, pp. 52-59, June 2010.

[2] G. H. Smith, D. Novak and Z. Ahmed, "Overcoming ChromaticDispersion Effects in Fiber-Wireless Systems Incorporating External Modulators," IEEE Transactions on Microwave Theory and Techniques, vol. 45, pp. 1410-1415, August 1997.

[3] M. Attygalle, C. Lim and A. Nirmalathas, "Extending Optical Transmission Distance in Fibre Wireless Links Using Passive Filtering in Conjunction with Optimized Modulation," IEEE Journal of Lightwave Technology, vol. 24, p. 1703 1709, April 2006.

[4] A. Nirmalathas, D. Novak, C. Lim and R. B. Waterhouse, "Wavelength Reuse in WDM Optical Interface of a Millimeter-Wave Fibre WirelessAntenna Base Stations," IEEE Transactions on Microwave Theory and Technology, vol. 49, pp. 2006 - 2009, October 2001.

[5] L. Chen, H. Wen and S. C. Wen, "A Radio-Over Fibre System With a Novel Scheme for Millimeter-Wave Generation and Wavelength Reuse for Up-link Connection," IEEE Photonics Technology Letters, vol. 18, pp. 2056 - 2058, October 2006.

[6] H-C. Ji, H. Kim and Y. C. Chung, "Cost-Effective Radio-Over-Fiber Systems Employing Phase-Modulated Downlink and Intensity-Modulated Uplink," (San Diego), March 2009.

[7] L. Chen, Y. Shao, X. Lei, H. Wen and S. Wen, "A Novel Radio-OverFiber System With Wavelength Reuse for Upstream Data Connection," IEEE Photonics Technology Letters, vol. 19, pp. 387 - 389, March 2007.

[8] J. Yu and G-K. Chang, "A Novel Technique for Optical Label and Payload Generation and Multiplexing using Optical Carrier Suppression and Separation," IEEE Photonics Technology Letters, vol. 16, pp. 320 322, January 2004.

[9] O. Akanbi, J. Yu and G-K. Chang, "A New Scheme for Bidirectional WDM-PON Using Upstream and Downstream Channels Generated by Optical Carrier Suppression and Separation Technique," IEEE Photonics Technology Letters, vol. 18, pp. 340 - 342, January 2006.

[10] J. Ma, X. Xin, C. Yu, J. Yu X. Sang and J. Zeng, "Millimeter-Wave Optical Subcarrier Generation by Using an External Modulator and Optical Carrier Suppression," International Conference on Transparent Optical Networks, pp. 273 - 276, July 2007.

[11] J. L. Corral and J. Marti and J. M. Fuster, "General Expressions for IM/DD Dispersive Analog Optical Links With External Modulation or Optical Up-Conversion in a MachZehnder Electro-Optical Modulator," IEEE Transaction on Microwave Theory and Techniques, vol. 49, pp. 1968-1976, October 2001.

[12] H. Schmuck, "Comparison of Optical Millimeter-Wave Concepts With Regard to Chromatic Dispersion," Electronics Letters, vol. 31, pp. 1848 - 1849, October 1995.

[13] W. H. Chen and W. I. Way, "Multichannel Single-Sideband SCM/DWDM Transmission Systems," IEEE Journal of Lightwave Technology, vol. 22, pp. 1679-1693, July 2004.

[14] J. G. Proakis, "Digital Communications," McGraw-Hill International, 4th Edition, August 2000.

[15] X. Huang, L. Zhang, M. Zhang, and P. Ye, "Impact of Nonlinear Phase Noise on Direct-Detection DQPSK WDM Systems," IEEE Photonics Technology Letters, vol. 17, pp. 1423 - 1425, July 2005.

[16] T-S. Cho and K. Kim, "Optimization of Radio-On-Fiber Systems Employing ODSB Signals by Utilizing a Dual-Electrode Mach-Zhender Modulator Against IM3," IEEE Photonics Technology Letters, vol. 18, pp. 1076-1078, May 2006. 